\title{
THE SCATTERING OF POSITRONS AND POSITRONIUM BY ATOMS IN THE CLOSE-COUPLING APPROXIMATION
}

\author{
R.N. HEWITT \\ Physique Théorique, Université Libre de Bruxelles, Campus Plaine CP 227 \\ Boulevard du Triomphe, 1050 Bruxellès, Belgium

\section{C.J. NOBLE}

DRAL Daresbury Laboratory, Daresbury, Warrington, WA4 4AD, U.K.

AND B.H. BRANSDEN

Department of Physics, University of Durham, South Road, Durham, DH1 3LE, U.K.

We present some recent results obtained within the close-coupling approximation for both positron and positronium scattering by atoms. We begin by discussing a generalization of the method of Vincent and Phatak to study the formation of $\mathrm{Ps}^{-}$in $\mathrm{Ps}$ collisions with $\mathrm{H}(1 s)$ in a simplified model. The important result of this calculation is that the overall Coulomb interaction which is present in the rearrangement channel is treated exactly. We also present results for positronium formation and ionization for positron scattering by argon atoms. The present calculations are for transitions from the $\operatorname{Ar}\left(3 s^{2}\right)$ subshell and show the importance of the coupling to the continuum.

PACS numbers: $36.10 .-k, 34.70 .+\mathrm{e}$

\section{Introduction}

The investigation of positron scattering by atoms has been the subject of an increasing number of theoretical investigations during the past decade. These have complemented the experimental activity in this field. The Detroit group have reported total cross-sections [1, 2] and positronium formation cross-sections [3] in the case of alkali atom targets. Positronium formation cross-sections have also been obtained in positron scattering by argon atoms [3]. The University College group in London is presently conducting experiments to investigate positronium-atom scattering [4]. 
We are interested in developing techniques to study both positron and positronium scattering by atomic targets. We have developed methods for obtaining the cross-sections for positron scattering by atoms by converting the usual coupled integro-differential equations into coupled integral equations of the LippmannSchwinger form in a momentum space representation. We have implemented these equations assuming that the overall interaction in each channel decreases faster than a potential of Coulomb form. The momentum space formulation has previously been successfully applied to treat the rearrangement process in positron-atom scattering $[5,6]$. The basis of our present method has been described in a series of papers [7, 8]. A novel feature of our present method is the use of Gaussian type expansions for the radial wave functions of the atomic orbitals.

In the case of the alkali atoms, $\mathrm{Na}$ and $\mathrm{K}$, very good agreement has been obtained with the experimental data of the Detroit group [1, 2] for both total measured cross-sections and total positronium formation cross-sections. We have also performed calculations, for positron scattering by helium atoms, within the single-active electron approximation [9]. Our results were in agreement with the experimental total cross-sections for the formation of positronium. Although we have successfully solved the Lippmann-Schwinger equations in the momentum space an important class of reactions cannot be treated immediately by the previous method. A reaction of the type

$$
\mathrm{A}+\mathrm{B} \rightarrow \mathrm{A}^{+}+\mathrm{B}^{-}
$$

results in an overall Coulomb interaction in the final rearrangement channel. The existence of such an interaction in either the initial or final channels of a reaction is a significant complication for the momentum space approach. It can be overcome by the use of distorted wave Green's functions which necessitates a sum over bound states in the case of an attractive Coulomb potential [10]. We have taken a different approach and generalized the method of Vincent and Phatak [11]. For a numerical demonstration we have chosen the formation of the negative positronium ion in collisions between positronium and hydrogen. We subsequently describe some new results for positronium formation and ionization in positron collisions with argon. In the case of argon we believe our results to be the first to include the simultaneous effects of coupling to the continuum and positronium formation. We are not aware of any previous close-coupled calculations for Ps $+\mathrm{H}$ collisions. In Sec. 2 we briefly outline some details of the numerical methods we have employed. Our results are described in Sec. 3 and our conclusions in Sec. 4.

\section{Numerical methods}

We begin by briefly describing our treatment of the positron-argon scattering system and refer to our previous papers for further details [7-9]. We treat the argon atom within the framework of the single-active electron approximation, i.e. we consider that a single-active electron in the $\operatorname{Ar}\left(3 s^{2}\right)$ subshell interacts with the ionic core of the atom via a model potential of the form

$$
V_{\mathrm{e}}(r)=-\frac{1}{r}-\exp (-a r)\left(\frac{Z-1}{r}\right)-c \exp (-b r),
$$

where $Z$ is the nuclear charge. The target Hamiltonian is now diagonalized on a basis of Gaussian type orbitals. The values of the parameters $a, b$ and $c$ are 
determined by requiring that the overlap between the resulting $\operatorname{Ar}(3 s)$ state and the corresponding Hartree-Fock state of Clementi and Roetti [12] is maximized. The final overlap value was 0.9991 corresponding to a binding energy of 1.028 a.u.

The potential between the incident positron and the ionic core was constructed by fitting a potential of the form

$$
V_{\mathrm{p}}(x)=-\frac{1}{x}-\exp (-d x)\left(\frac{Z-1}{x}\right)-g \exp (-f x)
$$

to the static potential obtained by averaging over the motion of the target ion electrons. The target wave function is the antisymmetrized Hartree-Fock wave function [12]. Our expansion basis for the positronium centre is obtained by diagonalizing a large Gaussian type basis to obtain atomic orbitals with negative and positive energy expectation values. The total flux into the positive energy orbitals (pseudostates) is taken to provide an estimate of the ionization cross-section. The present positronium centre basis consists of the exact $\operatorname{Ps}(1 s, 2 s, 2 p)$ eigenstates augmented by four $l=0$ and four $l=1$ pseudostates. The total basis set therefore consists of twelve atomic states.

Our methods for obtaining the numerical solution of the Lippmann-Schwinger equations in momentum space and the treatment of the singularity in the integrands has been discussed previously [13]. The presence of the two equivalent electrons in the $\operatorname{Ar}\left(3 s^{2}\right)$ subshell is accounted for by applying the independent particle model approximation [14]. The single electron transition cross-sections are obtained by combining the probabilities for an electron making a transition to a final state $f, p_{\mathrm{f}}$, and that for it to remain in the initial state $i, p_{\mathrm{i}}$, to obtain a final probability, $P$, of the form

$$
P=2 p_{\mathrm{i}} p_{\mathrm{f}} .
$$

The cross-section is then readily found.

We now describe our model for the Ps $+\mathrm{H}$ collision system. Within the limitations of the single-active electron approximation we can only include the rearrangement channel

$$
\mathrm{Ps}+\mathrm{H} \rightarrow \mathrm{H}^{+}+\mathrm{Ps}^{-} \text {, }
$$

which has a threshold at $13.278 \mathrm{eV}$. Subsequently the other possible rearrangement channel

$$
\mathrm{Ps}+\mathrm{II} \rightarrow \mathrm{e}^{+}+\mathrm{H}^{-}
$$

with a threshold at $6.03 \mathrm{eV}$, and the other breakup channels are neglected. The Ps atom is considered to be a structureless particle. The model potential representing the interaction between the active electron and the Ps core is taken to be the short range term of the semi-empirical potential discussed in [15]. This has the form

$$
V(s)=-0.4 \exp (-0.4589 s) \text {. }
$$

The diagonalization procedure produced a $\mathrm{Ps}^{-}$ground state with a binding energy of $-0.301 \mathrm{eV}$. The interaction between the proton and Ps, in this simplified model, is simply the static potential which is zero.

Our model is intended as a preliminary investigation of the Vincent-Phatak method applied to positronium scattering. Despite its simplicity it can be extended 
to energies close to the threshold (unlike presumably the distorted wave Born approximation (DWBA)). In the two-state approximation the total wave function is expanded as

$$
\Psi=\phi_{1 s}(\boldsymbol{r}) F(\boldsymbol{x})+\chi_{f}(s) G(\rho) .
$$

$\phi_{1 s}$ and $\chi_{f}$ are the internal wave functions of the hydrogen atom and $\mathrm{Ps}^{-}$ion respectively and $F$ and $G$ are the scattering wave functions. In our generalization of the Vincent-Phatak method we divide configuration space into an inner and outer region, defined by fixed values of the radial scattering coordinates. We assume that in each channel there exists a fixed radial distance $R_{\mathrm{C}}$ beyond which the interaction has effectively reached its asymptotic form. In each channel we divide the potential into short- and long-range contributions

$$
V \equiv V^{\mathrm{s}}+V^{\mathrm{L}}
$$

and in each channel $i$ :

$$
\begin{aligned}
& V_{i}^{\mathrm{S}}=V_{i} \theta\left(R_{\mathrm{C}}-\rho\right), \\
& V_{i}^{\mathrm{L}}=V_{i} \theta\left(\rho-R_{\mathrm{C}}\right) \approx V_{\mathrm{C}}(\rho) \theta\left(\rho-R_{\mathrm{C}}\right),
\end{aligned}
$$

where $V_{\mathrm{C}}$ is the point Coulomb interaction and $\theta$ is the Heaviside step function. The scattering $K$-matrix corresponding to the short-range potentials is obtained from the Lippmann-Schwinger equations by the standard procedures [13]. The solution of the complete scattering problem in the external region can be written in terms of matrices which include Ricatti-Bessel functions in the incident channel and Coulomb functions in the rearrangement channels. The $K$-matrix corresponding to the complete solution is determined by matching $R$-matrices at the boundary of the internal and external regions. The main difficulty associated with this method is in the choice of quadrature grids which are used to discretize the Lippmann-Schwinger equations for the short range cut-off potentials. In the present case up to two hundred quadrature points were needed in the rearrangement channel to obtain accurate results. The choice of grid was checked by accurately reproducing the correct solution for pure Coulomb elastic scattering in the rearrangement channel. These grids were subsequently used for the complete scattering problem.

\section{Results}

We begin by discussing the cross-sections obtained for positronium formation and ionization in positron-argon scattering. The present results are shown in Fig. 1 together with the data of McAlinden and Walters [16]. They employed the coupled-static approximation (CSA). The target was described by a fully antisymmetrized electronic wave function. However, they neglected the matrix elements which represent the exchange effects between the positronium and the target ion and refer to this as the truncated coupled-static approximation (TCSA). For a comparison we have also computed results within the same approximation in our model. There is excellent agreement between the present CSA, the present truncated CSA and the results of McAlinden and Walters above $80 \mathrm{eV}$. Below this energy the present results are significantly higher. The effect of the coupling with 


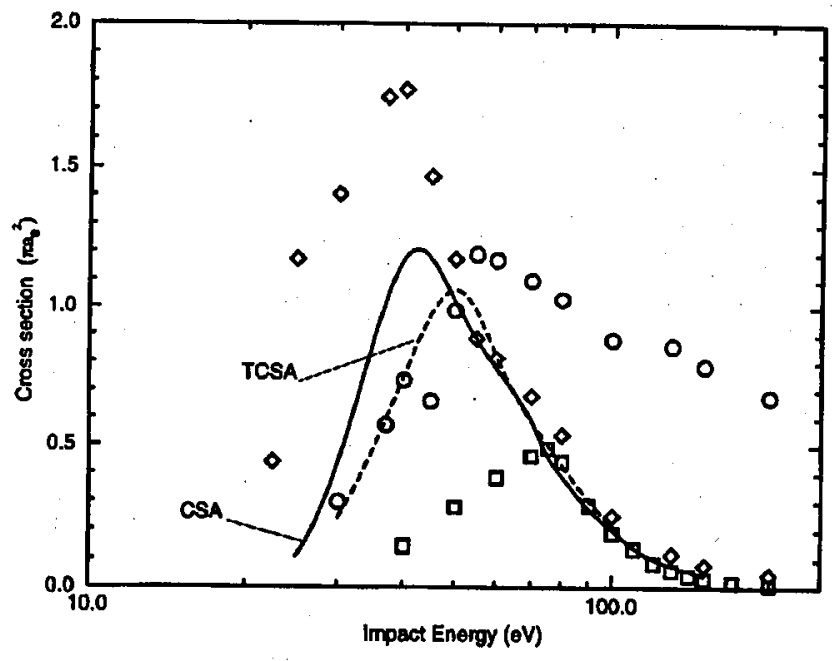

Fig. 1. Positronium formation and ionization cross-sections (in $\pi a_{0}^{2}$ ) versus the incident energy (in $\mathrm{eV}$ ) for positron collisions with argon atoms. Solid line: present $\operatorname{Ps}(1 s)$ from the CSA; dashed line: present $\operatorname{Ps}(1 s)$ from the truncated CSA; diamonds: present total for $\operatorname{Ps}(1 s, 2 s, 2 p)$ from the twelve state CCA; circles: present ionization total from the twelve state CCA; squares: Ps(1s), truncated CSA, obtained from Fig. 2 in [16].

the continuum states is to increase the total for Ps formation, while the energy at which the maximum in the cross-section occurs remains relatively unchanged, being close to $40 \mathrm{eV}$.

The cross-section for ionization reaches a maximum at about $60 \mathrm{eV}$. The secondary maximum close to $30 \mathrm{eV}$ may be due to the presence of the unphysical pseudostate thresholds. For impact energies above $50 \mathrm{eV}$ the ionization channels become increasingly dominant. It is interesting to note that total ionization cross-section measurements show a maximum for impact energies close to $60 \mathrm{eV}$ [17], while the total Ps formation cross-section measurements show a maximum between $20-30 \mathrm{eV}$ [3].

We now discuss the results concerning the formation of the $\mathrm{Ps}^{-}$ion in $\mathrm{Ps}+\mathrm{H}$ collisions. We are aware of three other estimates for this cross-section, which are displayed in Fig. 2. The first was within the first Born approximation (FBA) [18]: The classical trajectory Monte Carlo method (CTMC) and a distorted wave Born approximation have also been applied to this system [19]. The Coulomb attraction in the rearrangement channel leads to a finite cross-section at threshold, which accounts for the behaviour of the present cross-sections at energies below $15 \mathrm{eV}$. The Born approximation does not reproduce this feature. We have also computed a distorted wave approximation (DWA) by neglecting the coupling of the initial channel to the rearrangement channel. This calculation differs significantly from the present close-coupling approximation (CCA) only for impact energies below about $40 \mathrm{eV}$. Compared to the present model, additional polarization terms were included in the $\mathrm{e}^{-}-\mathrm{Ps}$ and $\mathrm{H}^{+}-\mathrm{Ps}$ potentials in the DWBA [19]. The form of 


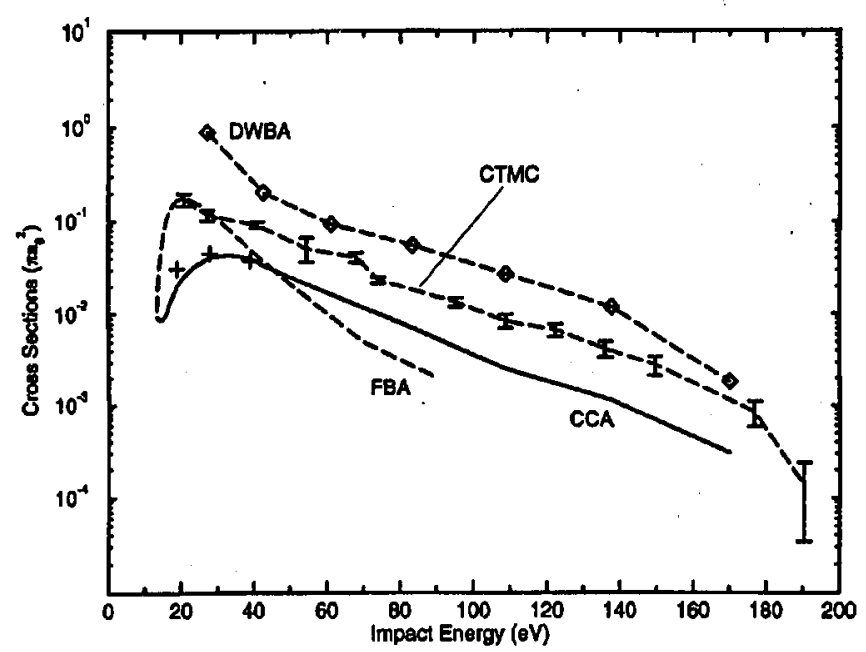

Fig. 2. Cross-sections (in $\pi a_{0}^{2}$ ) for the formation of the positronium negative ion versus the incident energy (in eV) for positronium collisions with hydrogen atoms. Solid line: present two-state close-coupling (CCA) using the Vincent-Phatak method to treat the final-state Coulomb interaction; plus: distorted wave approximation neglecting coupling between initial and final channels; diamonds: distorted wave Born approximation [19]; dash line: first Born approximation [18]; dash line with error bars: CTMC approximation [19].

the DWBA also differs from the present DWA. It is therefore not unexpected to observe that their cross-sections are significantly larger than the present results given that the rearrangement cross-sections are known to often be very sensitive to the precise details of the model employed.

\section{Conclusions}

We have presented a generalization of the method of Vincent and Phatak to obtain the rearrangement cross-sections for a system with an overall Coulomb potential present in the final channel. We have shown the method is numerically applicable in the particular case of Ps $+\mathrm{H}$ collisions. It will be necessary to remove the one active electron approximation and include polarization effects to obtain more accurate results and we are now pursuing this. The present results obtained for $\mathrm{e}^{+}+\operatorname{Ar}\left(3 s^{2}\right)$ scattering within the independent electron model are very encouraging. There is agreement with existing results for impact energies above $80 \mathrm{eV}$ for the $\mathrm{Ps}(1 \mathrm{~s})$ formation cross-section. The effect of the coupling to the continuum has also been demonstrated to be important for impact energies below about $60 \mathrm{eV}$, and an estimate of the ionization cross-section obtained. The extension of the method to include transitions from the $\operatorname{Ar}\left(3 p^{6}\right)$ subshell is now being undertaken in order to allow a comparison with the experimental data to be made. 


\section{References}

[1] S.P. Parikh, W.E. Kauppila, C.K. Kwan, R.A. Lukaszew, D. Przybyla, T.S. Stein, Z. Zhou, Phys. Rev. A 47, 1535 (1993).

[2] C.K. Kwan, W.E. Kauppila, R.A. Lukaszew, S.P. Parikh, T.S. Stein, Y.J. Wan, M.S. Dababneh, Phys. Rev. A 44, 1620 (1991).

[3] T.S. Stein, W.E. Kauppila, C.K. Kwan, S.P. Parikh, S. Zhou, Hyperfine Interact. 73, 53 (1992).

[4] M. Charlton, G. Laricchia, Comm. At. Mol. Phys. 26, 253 (1991).

[5] A.S. Ghosh, D. Basu, Ind. J. Phys. 47, 765 (1973).

[6] M. Basu, M. Mukherjee, A.S. Ghosh, J. Phys. B, At. Mol. Opt. Phys. 22, 2195 (1989).

[7] R.N. Hewitt, C.J. Noble, B.H. Bransden, J. Phys. B, At. Mol. Opt. Phys. 23, 4185 (1990).

[8] R.N. Hewitt, C.J. Noble, B.H. Bransden, J. Phys. B, At. Mol. Opt. Phys. 26, 3661 (1993).

[9] R.N. Hewitt, C.J. Noble, B.H. Bransden, J. Phys. B, At. Mol. Opt. Phys. 25, 557 (1992).

[10] I. Bray, Phys. Rev. A 49, 1066 (1994).

[11] C.M. Vincent, S.C. Phatak, Phys. Rev. C 10, 391 (1974).

[12] E. Clementi, C. Roetti, At. Data Nucl. Dala Tables 14, 177 (1974).

[13] B.H. Bransden, C.J. Noble, R.N. Hewitt, J. Phys. B, At. Mol. Opt. Phys. 26, 2487 (1983).

[14] J.H. McGuire, L. Weaver, Phys. Rev. A 16, 41 (1977).

[15] A.M. Ermolaev, C.R. Mandal, J. Phys. B, At. Mol. Opt. Phys. 21, 2077 (1988).

[16] M.T. McAlinden, H.R.J. Walters, Hyperfine Inleract. 73, 65 (1992).

[17] H. Knudsen, L. Brun-Nielsen, M. Charlton, M.R. Poulsen, J. Phys. B 23, 3955 (1990).

[18] A. Stancanelli, G. Ferrante, Nuovo Cimento LXVIII, 137 (1969).

[19] A.M. Ermolaev, B.H. Bransden, C.R. Mandal, J. Phys. B, At. Mol. Opt. Phys. 22, 3717 (1989). 